\title{
A rare case of urinary tract infection with Burkholderia cepacia in a male child
}

\author{
Iulia Armean', Lorena Elena Melit ${ }^{1,2}$, Carmen Duicu, ${ }^{1,2}$ \\ ${ }^{1}$ Pediatrics Clinic, Emergency Clinical County Hospital, Tg. Mures \\ ${ }^{2}$ University of Medicine and Pharmacy, Tg. Mures
}

\begin{abstract}
Introduction. Burkholderia cepacia is an aerobic, glucose-non-fermenting, gram negative bacillus that mainly affects immunocompromised and hospitalized patients. Urinary tract infections (UTI) caused by this aggressive pathogen are rarely described in the literature. Patients who associate predisposing risk factors such as post renal transplant, vesico-ureteral reflux (VUR), neurogenic bladder, bladder irrigation, or use of contaminated medical devices, are susceptible to B. cepacia UTI.

Case presentation. We report the case of a 5-year-old boy, admitted in the Pediatrics Clinic, for fever, cloudy and malodorous urine. His personal history revealed surgery for myelomeningocele, ventriculo-peritoneal shunt for congenital hydrocephalus, lower limb paraplegia, and bilateral hydronephrosis grade III, neurogenic bladder and recurrent UTI. The laboratory tests performed on the day of admission revealed increased inflammatory biomarkers, leukocytosis and anemia. The urine test revealed pyuria and the urine culture, obtained by bladder catheterization, was positive for multiple drug resistant $B$. cepacia. Antibiotic therapy was initiated with Meronem and symptomatic drugs. After 10 days of hospitalization the patient was discharged in general good status with the recommendations of chemoprophylaxis.

Conclusions. Despite the very low incidence of $B$. cepacia UTI in children, we present the case of a patient who associate predisposing factors such as neurogenic bladder, hydronephrosis and recurrent UTI who lead to an aggressive infection with this opportunistic pathogen.
\end{abstract}

Keywords: urinary tract infection, children, Burkholderia cepacia

\section{INTRODUCTION}

Urinary tract infection (UTI) represents a pathology with bacterial etiology, frequently encountered among children that can associate severe complications such as renal scars or chronic renal failure (1). In case of children, it is the most frequent bacterial infection after otitis media (2). UTI is defined as significant bacteriuria (growth of a single pathogen: at least $10^{4} \mathrm{CFU}$ for catheter specimens and at least $10^{5} \mathrm{CFU}$ for midstream cleancatch specimens) or $5 \times 10^{4} \mathrm{CFU}$ and significant pyuria in a patient with fever or other clinical symptoms $(3,4)$. Recurrent UTIs are defined as repeated infections with different pathogen agent, while recidivated UTIs represent repeated infections with the same pathogen (5). UTI is diagnosed in approximately $5 \%$ of the infants that come in the emergency rooms complaining of fever without any obvious cause (1). The prevalence and incidence of ITU among children vary depending on the age, ethnicity and the presence or absence of circumcision (6). Until the age of 6 month, boys are more susceptible to developing ITU in comparison to girls, but the incidence of this infection remains substantially higher among females (5). Until the age of 6 years, at least $2 \%$ of the boys and $7 \%$ of the girls presents an episode of UTI (5), the Caucasians being more susceptible to than the black one $(5,7)$. Circumcision can have a protective effect, decreas- 
ing by $87 \%$ the risk of infection, even more important in case of boys that present recurrent UTIs, posterior urethral valve or high grade vesicoureteral reflux $(5,8)$. Among the pathogen agents, Echerichia Coli represents the most frequent bacteria causing between $85 \%$ and $90 \%$ of all cystitis and pyelonephritis episodes in children (2), but Klebsiella, Proteus, Enterobacter, Staphylococcus saprophiticus and Enerococcus can also be encountered (1). Community-acquired UTIs differentiate from the nosocomial ones through the type of germs that affect patient with prolonged hospitalizations, these associating many times multiple comorbidities, recurrent UTI and a history of multiple antibiotic treatments (9). Among these agents is also encountered Burkholderia Cepacia (B. Cepacia), aerobe bacillus, gram negative that does not ferment glucose, with multidrug resistance and to antiseptic agents that affects mainly immunocompromised patients (10-12). B. Cepacia causes most frequently respiratory tract infections, septic arthritis and UTIs (13). The specialty literature reports also cases of colonization with $B$. cepacia in drug addicted patients that present endocarditis or in those with mechanic cardiac valves, ocular infections after surgical interventions and central nervous system infections (12). B. cepacia represents an important cause of morbidity and mortality among patients with prolonged hospitalizations in intensive care units or oncology departments (14). Chronic infections with $B$. cepacia are difficult to be eradicated possibly due to its capacity to cause invasive diseases and due to its increased level of resistance to antibiotics (15). Patients with cystic fibrosis and those with chronic granulomatous disease are the most predisposed to develop infections caused by B. cepacia due to their immune deficiency $(13,16)$. Regarding the kidney, the predisposing factors for the infections with $B$. cepacia are: vesico-ureteral reflux, neurogenic urinary bladder, urinary bladder irrigations, renal transplantations and the use of contaminated medical devices $(11,17)$. In children, UTI caused by B. cepacia is very rarely reported (11).

The aim of this case report is to highlight the very low frequency of UTI caused by B. cepacia in a patient with multiple risk factors for developing this infection and other comorbidities.

\section{CASE REPORT}

\section{Reasons of admission}

We report the case of a 5 years and 3 months old male child, admitted in our clinic for the following complaints: initially low fever for 2 days, which increased afterwards associating bad smelling, turbid urine. The personal pathological history revealed a surgical intervention for myelomeningocele and congenital hydrocephaly for which he underwent a ventriculo-peritoneal shunt during the neonatal period, flask paraplegy of the inferior limbs, weight and height deficit, bilateral $3^{\text {rd }}$ degree hydronephrosis, neurogenic urinary bladder and recidivate UTIs (former pathogenic agents: Klebsiella pneumoniae BLSE, Pseudomonas, Enterococcus faecalis).

\section{Clinical exam}

At the time of admission, the clinical exam revealed the following pathological elements: fever $38.8^{\circ} \mathrm{C}$, influenced general status, bilateral lumbar and sacral post-surgical scar and at the level of the right abdominal side, right latero-cervical and right anterior hemithorax palpable peritoneal shunt, hypotonia of the inferior limbs with absent osteotendinous reflexes, defecation and micturition reflexes not acquired, weight $14 \mathrm{~kg}$.

\section{Diagnostic assessment}

Taking into account the personal pathological history, we raised the suspicion of a UTI. Therefore, the initial CBC count pointed out: hemoglobin $11 \mathrm{~g} / \mathrm{dl}$, hematocrit $32.8 \%$, medium erythrocyte volume $74.5 \mathrm{fL}$, leukocytes $18,510 / \mu 1$, neutrophils $63.6 \%$, lymphocytes $25.3 \%$, platelets $286,000 / \mu 1$; elevated inflammatory biomarkers: CRP $74.7 \mathrm{mg} /$ $\mathrm{dl}$, ESR $105 \mathrm{~mm} / \mathrm{h}$. The urinary exam revealed: leukocytes over $500 / \mu 1$ and erythrocytes $50 / \mu 1$. The urine culture, obtained by urinary bladder catheterization, was positive for Burkholderia cepacia > $100,000 \mathrm{CFU} / \mathrm{ml}$ urine, and the antibiogram showed resistance to Amikacin, Cefepime, Ciprofloxacin, Colistin, Gentamicin, Imipenem, Piperacillin, Piperacillin/Tazobactam, Ticarcillin; intermediary results for Ceftazidime and sensitivity to Meropenem, Minocycline and Trimethoprim/Sulfamethoxazole.

Abdominal ultrasound pointed out: normal size kidneys, bilaterally reduced parenchymal index, 
lack of corticomedullary differentiation, increased cortical echogenicity, bilaterally grade III ureterohydronephrosis, irregular, thickened bladder wall, bladder diverticulum. Retro cystography and renal scintigraphy were performed.

\section{Therapeutic assessment}

As a result of establishing the diagnosis of high B. cepacia UTI, we initiated antibiotic treatment according to the antibiogram with Meropenem and symptomatic drugs, the evolution being favorable. After 10 days of admission, the patients was discharged with good general status, chemoprophylaxis recommendations with trimethoprim/sulfamethoxazole and intermittent catheterizations 5 times per day.

\section{DISCUSSIONS}

Early diagnosis and treatment of acute ITU is of vital importance because most of the time children develop pyelonephritis, which may have serious repercussions on the renal parenchyma (1). ITU may be the first sign in $30 \%$ of children who associate urinary tract abnormalities (4).

In renal transplant patients, UTI is the most common infectious complication, contributing significantly to increased mortality and morbidity among these patients (17).

Structural abnormalities, urological procedures, stents placement, colonization of urinary catheters or bladder irrigation with contaminated fluids are well-known risk factors. They can represent nidus for bacterial growth, leading to infection and also predisposing to recurrence (11).

In some cases, rare opportunistic pathogens, which are most often considered contaminants, can cause infections. B. cepacia is not a common pathogen for urogenital tract infections, which is often inseminated during surgical maneuvers or catheterization (17), thus there are few studies on the ITU characteristics caused by B. cepacia. However, patients who develop this type of infection often associate predisposing factors such as kidney transplantation, prolonged urinary bladder catheterization, history of ureterocystoscopy, and kidney malformations (11). Similarly, the abovementioned patient associated risk factors such as: bilateral hydronephrosis, neurogenic bladder, multiple catheterization, and recurrent ITU. B. cepacia, a bacterium that most often affects immunocompromised patients, lives in moist environments, cases of infections associated with contaminated nebulizers, mouthwash, body cream, and water storage devices being described. Furthermore, in hospitals, it is also disseminated through the equipment used to support the respiratory function, disinfectant solutions and blood analyzers $(11,18)$. This pathogen associates low mortality and morbidity, although it has an inherent high resistance to multiple antimicrobial agents and antiseptic agents (18). Thus, recent studies have shown that B. cepacia expresses, in most cases, sensitivity to meropenem, piperacillin-tazobactam and trimethoprimsulfamethoxazole and $100 \%$ resistance to amikacin and colistin (11). Due to this multi-drug resistance, in some cases combinations of antimicrobials, or even surgical treatment is required (15). Multi-drug resistance was also observed in our patient, the antibiogram pointing out sensitivity to meropenem and trimethoprim/sulfamethoxazole, as reported in the literature.

\section{CONCLUSIONS}

Although B. cepacia usually causes respiratory tract infections, it can also lead to UTI in children. The presence of risk factors is mandatory in the development of this infection, our patient also associating multiple comorbidities. Another characteristic of this pathogen is the multi-drug resistance, also proven in the case presented above.

\section{REFERENCES}

1. Gorczyca D., Augustyniak D., Basiewicz W.B., Karnas K.W. Serum and Urinary MIP-1 $\alpha$ and IP-10 Levels in Children with Urinary Tract Infections. Adv Clin Exp Med. 2014; 23:933-938.

2. Becknell B., Schober M., Korbel L., Spencer J.D. The Diagnosis, Evaluation and Treatment of Acute and Recurrent Pediatric Urinary Tract Infections. Expert Rev Anti Infect Ther. 2015; 13:81-90.

3. NICE Guideline. Urinary tract infection in under 16s: Diagnosis and management (CG54). Update 2017.

4. Stein R., Dogan H.S., Hoebeke P., Kocvara R., Nijman R.J., Radmajr C. et al. Urinary tract infections in children: EAU/ESPU guidelines. EurUrol. 2015; 67:546-58. 
5. Larcombe J. Urinary Tract Infection in Children. American Family Physician. 2010; 82:1252-1254.

6. Hillary L., Schmidt B. Work up of Pediatric Urinary Tract Infection. Urol Clin North Am. 2015; 42:519-526.

7. Chen L., Baker M.D. Racial and ethnic differences in the rates of urinary tract infections in febrile infants in the emergency department. Pediatr Emerg Care. 2006; 22:485-487.

8. Mukherjee S., Joshi A., Carroll D. What is the effect of circumcision on risk of urinary tract infection in boys with posterior urethral valves? J Pediatr Surg. 2009; 44:417-421.

9. Nimri L., Sulaiman M., Bani O.H. Community-acquired urinary tract infections caused by Burkholderia cepacia complex in patients with no underlying risk factor. JMM Case Reports. 2017;4.

10. Murat D., Ozlem G.T., Busra E.S., Firdevs A., Dilek A. Nosocomial Burkholderia cepacia infections in a Turkish university hospital: A five-year surveillance. J Infect Dev Ctries. 2009; 3:273-277.

11. Lee K.W., Lee S.T., Cho H. Burkholderia cepacia Causing Nosocomial Urinary Tract Infection in Children. Child Kidney Dis. 2015; 19:143-147.

12. Kim K.Y., Yong D., Lee K., Kim H.S., Kim D.S. Burkholderia Sepsis in Children as a Hospital-Acquired Infection. Yonsei Med J. 2016 Jan; 57:97-102.
13. Gautam V., Singhal L., Ray P. Burkholderia cepacia complex: Beyond pseudomonas and acinetobacter. Indian J Med Microbiol. $2011 ; 29: 4-12$

14. Mukhopadhyay C., Bhargava A., Ayyagari A. Two Novel Clinical Presentations of Burkholderia cepacia Infection. J Clin Microbiol. 2004; 42:3904-3905.

15. Messiaen A.S., Nelis H., Coenye T. Investigating the role of matrix components in protection of Burkholderia cepacia complex biofilms against tobramycin. J Cyst Fibros. 2014: 13:56-62.

16. Vining M., Sharma N., Guill M. Atypical presentation of chronic granulomatous disease with Burkholderia cepacia. BMJ Case Rep. 2014, doi:10.1136/bcr-2013-201524

17. Zeeshan M., Aziz T., Naqvi F. Recurrent urinary tract infection by Burkholderia cepacia in a live related renal transplant recipient. J Pak Med Assoc. 2012; 62:496-8.

18. Lerma A.F., Maull E., Terradas R., Segura C., Planells I. Moisturizing body milk as a reservoir of Burkholderia cepacia: Outbreak of nosocomial infection in a multidisciplinary intensive care unit. Critical Care. 2008; 12:1-6.

19. White B. Diagnosis and Treatment of Urinary Tract Infections in Children. Am Fam Physician. 2011; 83:409-415 